\title{
Dissociation of the Role of Infralimbic Cortex in Learning and Consolidation of Extinction of Recent and Remote Aversion Memory
}

\author{
Walaa Awad', Guillaume Ferreira ${ }^{2,3,4}$ and Mouna Maroun*,I,4 \\ 'Sagol Department of Neurobiology, Faculty of Natural Sciences, University of Haifa, Haifa, Israel; ${ }^{2}$ Nutrition and Integrative Neurobiology, \\ INRA UMR 1286, Bordeaux, France; ${ }^{3}$ Université de Bordeaux, Bordeaux, France
}

\begin{abstract}
Medial prefrontal circuits have been reported to undergo a major reorganization over time and gradually take a more important role for remote emotional memories such as contextual fear memory or food aversion memory. The medial prefrontal cortex, and specifically its ventral subregion, the infralimbic cortex $(\mathrm{IL})$, was also reported to be critical for recent memory extinction of contextual fear conditioning and conditioned odor aversion. However, its exact role in the extinction of remotely acquired information is still not clear. Using postretrieval blockade of protein synthesis or inactivation of the IL, we showed that the IL is similarly required for extinction consolidation of recent and remote fear memory. However, in odor aversion memory, the IL was only involved in extinction consolidation of recent, but not remote, memory. In contrast, only remote retrieval of aversion memory induced c-Fos activation in the IL and preretrieval inactivation of the IL with lidocaine impaired subsequent extinction of remote but not recent memory, indicating IL is necessary for extinction learning of remote aversion memory. In contrast to the effects in odor aversion, our data show that the involvement of the IL in the consolidation of fear extinction does not depend on the memory age. More importantly, our data indicate that the IL is implicated in the extinction of fear and nonfear-based associations and suggest dissociation in the engagement of the IL in the learning and consolidation of food aversion extinction over time.

Neuropsychopharmacology (20I5) 40, 2566-2575; doi:I0.I038/npp.2015.I03; published online 27 May 2015
\end{abstract}

\section{INTRODUCTION}

Emotional memories that are formed following traumatic/ stressful events are engraved and can last for life time. These memories can be retrieved after several weeks/months (remote memory) and do not undergo weakening of the memory trace.

Studies on both humans and experimental animals show that remote memories are stored in cortical regions (Knowlton and Fanselow, 1998; Squire and Alvarez, 1995). In particular, medial prefrontal cortices (mPFCs) have been reported to undergo a major reorganization over time and gradually take a more important integrative role for remote emotional memories such as contextual fear memory (CFC; Einarsson et al, 2014; Frankland et al, 2004; Goshen et al, 2011; Restivo et al, 2009; Vetere et al, 2011) or food aversion memory (Ding et al, 2008; Frankland and Bontempi, 2005). The mPFC and specifically its ventral subregion, the infralimbic part (IL), was also reported to be critical for

* Correspondence: Professor M Maroun, Sagol Department of Neurobiology, Faculty of Natural Sciences, University of Haifa, Haifa 31905, Israel, Tel: 0097248288 634, Fax: 0097248240339 , E-mail: mouna.maroun@gmail.com

${ }^{4}$ The last two authors contributed equally to this work. Received 20 October 20 I4; revised I 8 March 2015; accepted 29 March 2015; accepted article preview online 15 April 2015 extinction of emotional memories as transient pharmacological manipulation of the IL altered extinction of recent memory of CFC (Kritman and Maroun, 2012; Laurent and Westbrook, 2009; Sierra-Mercado et al, 2010) and conditioned food aversion (Akirav et al, 2006a). Although substantial advances have been made in our understanding of the role of the IL in the extinction of recently acquired information, still its exact role in mediating extinction of remotely aversive memories is not well documented. This question is particularly relevant regarding recent evidences indicating behavioral and neurobiological differences between recent and remote memory extinction (Corcoran et al, 2013; Inda et al, 2011; Lopez et al, 2008). It is important to note that standard theory of systems memory consolidation postulates that the hippocampus has a time-limited role in the storage of memories, with a more important role of the mPFC in remote memories as was shown for CFC (Frankland and Bontempi, 2005; Knowlton and Fanselow, 1998; Squire and Alvarez, 1995). Even if recent evidence indicates that IL is similarly involved in extinction consolidation of both recent (1 day) and remote (14 days) fear memories (Rosas-Vidal et al, 2014), a hippocampaldependent memory task (Goshen et al, 2011; Zelikowsky et al, 2012), no study has yet investigated and compared the role played by the IL in extinction of recent and remote memory in conditioned food aversion, a nonfear-based 
association considered as a hippocampal-independent memory task (Ballarini et al, 2009; Cui et al, 2005; Ding et al, 2008; Josselyn et al, 2004). For this purpose, we used conditioned odor aversion (COA) that relies on the association between tasteless odorized water and malaise feeling that creates robust memory that we have previously shown to last for weeks (Chapuis et al, 2007; Desgranges et al, 2009; Sevelinges et al, 2009a, b).

Here, using local pharmacological challenges (blockade of protein synthesis or inactivation) and neuronal activity marker (c-Fos immunohistochemistry), we confirm that IL is required for extinction consolidation of recent ( 1 day) and remote (28 days) fear memory, but we show for the first time that IL is only involved in extinction consolidation of recent (2 days), but not remote (28 days), COA memory. Moreover, $\mathrm{IL}$ is necessary for extinction learning of remote, but not recent, COA memory, suggesting dissociation in the role played by IL in learning and consolidation of extinction depending on the age of COA memory.

\section{MATERIALS AND METHODS}

\section{Animals and Surgery}

The experiments were performed on male Sprague-Dawley rats (Harlan Laboratories, Jerusalem) weighing 200-280 g. The animals were housed in Plexiglas cages (five rats per cage) and were maintained on a free-feeding regimen with a 12-h light and 12-h dark schedule (0700 to $1900 \mathrm{~h})$. A week after arrival, the rats were anesthetized at $4.8 \mathrm{ml} / \mathrm{kg}$ with Equithesin $\left(2.12 \%(\mathrm{w} / \mathrm{v}) \mathrm{MgSO}_{4}, 10 \%(\mathrm{v} / \mathrm{v})\right.$ ethanol, $39.1 \%$ (v/v) propylene glycol, $0.98 \%(\mathrm{w} / \mathrm{v})$ sodium pentobarbital, and $4.2 \%(\mathrm{w} / \mathrm{v})$ chloral hydrate) and placed in a stereotaxic frame, with body temperature maintained at $37 \pm 0.5^{\circ} \mathrm{C}$. According to Paxinos and Watson (1998), the animals were implanted bilaterally with stainless steel guide cannulae (23 gauge) aimed at the IL (anteroposterior, $+3.2 \mathrm{~mm}$ relative to Bregma; lateral, $\pm 0.5 \mathrm{~mm}$ from midline; ventral, $-4.6 \mathrm{~mm}$ from Bregma). The cannulae were held in place with acrylic dental cement and secured with two skull screws. A stylus was placed in the guide cannula to prevent clogging. The animals were allowed 1 week to recuperate before being subjected to experimental procedures. The procedures were performed in strict accordance with the University of Haifa regulations and the US National Institutes of Health guidelines (NIH publication number 8023).

\section{Drugs and Infusion Procedure}

The anesthetic lidocaine hydrochloride (Sigma, France) was dissolved in sterile $0.9 \%$ saline solution to obtain a final concentration of $4 \%$. The dose of lidocaine used here induced a complete inhibition of neural activity within $5 \mathrm{~min}$ after infusion that lasts for 15-30 min (Boehnke and Rasmusson, 2001; Lomber, 1999; Tehovnik and Sommer, 1997). The protein synthesis inhibitor, anisomycin (Sigma), was dissolved in $1 \mathrm{M} \mathrm{HCl}$, adjusted to $\mathrm{pH} 7.2$ with $\mathrm{NaOH}$, and brought to final concentration of $100 \mu \mathrm{g} / \mu \mathrm{l}$ with sterile saline (Akirav et al, 2006a; Berman and Dudai, 2001; Eisenberg et al, 2003). Control rats were microinjected with the appropriate vehicle. For microinfusion, the stylus was removed from the guide cannula, and a 28-gauge injection cannula, extending $1.0 \mathrm{~mm}$ beyond the tip of the guide cannula, was inserted. The injection cannula was connected via PE20 tubing to a Hamilton microsyringe driven by a CMA/100 microinfusion pump (Hamilton, Switzerland). Microinfusion was performed bilaterally, delivering a $0.5-\mu \mathrm{l}$ volume over $1 \mathrm{~min}$. The injection cannula was left in position for an additional $1 \mathrm{~min}$ before withdrawal, to minimize dragging of the injected liquid along the injection tract.

\section{Behavior}

Contextual fear conditioning. The conditioning and its subsequent extinction protocols are based on our previous reports (Akirav et al, 2009; Kritman and Maroun, 2012; Lahoud and Maroun, 2013; Motanis and Maroun, 2010). The rats were placed in a conditioning chamber (Panlab, Spain) with floor grids, black methacrylate walls, and a transparent front door. For conditioning the animals received three footshocks delivered through the grids at intensity of $0.8 \mathrm{~mA}$ for $0.5 \mathrm{~s}$. The shocks were administered 90, 210, and $330 \mathrm{~s}$ after the animal was placed in the conditioning chamber. Thus, the conditioning session lasted $\sim 8 \mathrm{~min}$. The chamber was cleaned with $70 \%$ ethanol wipes and dried with paper towels after each rat. For the tests following conditioning, the rats were submitted to a 10-min nonreinforced test trial (placed in the chamber without receiving the electrical shock) every $24 \mathrm{~h}$ for 3 days (T1-T3), starting $24 \mathrm{~h}$ or 28 days after conditioning. An analog signal was generated by the animal's movement by means of a high-sensitivity weight-transducer system connected to the grid floor. The signal was transmitted through the load cell unit to the software module for offline analysis. Freezing, ie, the absence of all movement except for respiration (Blanchard and Blanchard, 1972; Kim et al, 1992), was quantified offline. Freezing over $5 \mathrm{~min}$ (retrieval) and $10 \mathrm{~min}$ (extinction) test was averaged and presented as percentage of the time the animal was in freezing during the test. The first 2 min served as acclimatization to the chamber in which the rat explored the context and the last 2 min served after the third shock were used to estimate the success of the conditioning.

Conditioned odor aversion. The protocol is based on our previous reports (Chapuis et al, 2007; Desgranges et al, 2009; Sevelinges et al, 2009a). In this paradigm, banana-scented solution (isoamyl acetate, Sigma, $(0.01 \% \mathrm{w} / \mathrm{v})$ ) was used as the conditioned stimulus (CS) and lithium chloride $(\mathrm{LiCl}$, $0.15 \mathrm{M}, 1 \%$ body weight, intraperitoneally (i.p.); Sigma) was used as a malaise-inducing agent (unconditioned stimulus (US)). The banana solution was chosen because previous studies indicated that its processing is mediated by odor, and not by any taste properties (Slotnick, et al, 1997). Indeed, anosmic rats were unable to reliably detect $0.1 \%$ bananascented water, whereas they performed as well as control for taste detection (Slotnick, et al, 1997). Furthermore, inactivation of the insular/gustatory cortex had no effect on COA, suggesting that the banana solution has no taste (Desgranges et al, 2009). Briefly, during the first 4 days of the behavioral experiments, rats were trained to get their daily water ration, once a day, for $20 \mathrm{~min}$, from two pipettes each containing $10 \mathrm{ml}$ of tap water. On day 5, which was the conditioning day, rats were allowed to drink $20 \mathrm{ml}$ of the banana solution (instead of water). At $40 \mathrm{~min}$ after the offset of the drinking 
period, they received an intraperitoneal injection of $\mathrm{LiCl}$. For the next day(s), the rats were presented for 20 min with two pipettes each containing $10 \mathrm{ml}$ of tap water. At 2 or 28 days after training, each rat was given two pipettes $(10 \mathrm{ml}$ each) of banana solution for $20 \mathrm{~min}$ and the amount of consumed solution was measured. Immediately after two pipettes of water, each containing $10 \mathrm{ml}$ were given for $20 \mathrm{~min}$ to the rats. For COA extinction, the test was repeated for 2 days. The conditioned aversion is presented as an aversive index, defined as $(\mathrm{ml}$ water $/(\mathrm{ml}$ water $+\mathrm{ml}$ banana $) \times 100)$ consumed in the test; hence, 50 indicates equal preference. Higher aversion index means higher preference of water over banana-scented solution.

\section{Histology}

On completion of the behavioral experiments, animals were anesthetized, $0.5 \mu \mathrm{l}$ of Indian ink was microinfused into the IL, and the cannula locations were examined under a light microscope, following Nissl staining. Figure 1 shows a schematic drawing of the cannulae placements in the IL (coronal view at position +3.20 and $+2.70 \mathrm{~mm}$ anterior to Bregma). Solid black circles indicate the locations. Animals that did not have the tip of their cannulae in the IL were excluded from analysis.

\section{Immunohistochemical Experiments: Neuronal Activity Marker}

Rats were killed with an i.p. injection of a lethal dose of pentobarbital sodium $(1 \mathrm{ml})$ occurring 90 min upon termination of a 20-min banana odor presentation performed 2 or 28 days after odor aversion conditioning (banana odor- $\mathrm{LiCl}$ pairing) or pseudoconditioning (banana odor- $\mathrm{NaCl}$ pairing). They were perfused transcardially with $0.1 \mathrm{M}$ phosphatebuffered saline (PBS, $\mathrm{pH}=7.4$ ) followed by $4 \%$ paraformaldehyde (PFA) in PBS. Brains were quickly removed and stored at $4{ }^{\circ} \mathrm{C}$ in a $4 \%$ PFA for $24 \mathrm{~h}$ to allow postfixation. The next day, they were submerged in a $30 \%$ sucrose solution at $4{ }^{\circ} \mathrm{C}$ for $48 \mathrm{~h}$ to allow cryoprotection. Finally brains were frozen in isopentane and stored at $-80^{\circ} \mathrm{C}$. Coronal sections of $40 \mu \mathrm{m}$ were cut from frontal cortex and incubated in PBS containing $0.1 \%$ bovine serum albumin (BSA) and $0.5 \%$ Triton (PBSBSA-T) to block nonspecific binding sites and to facilitate antibody penetration. The sections were also saturated with $0.3 \%$ hydrogen peroxide for $30 \mathrm{~min}$ to eliminate endogenous peroxydase activity. Sections were incubated with the primary anti-c-Fos antibody (anti-c-Fos rabbit polyclonal antibody, $1: 1000$ diluted in PBS-BSA-T; Santa Cruz Biotechnology) for $24 \mathrm{~h}$ at room temperature, then incubated for $2 \mathrm{~h}$ with the biotinylated secondary antibody at room temperature (goat anti-rabbit IgG, diluted 1:2000 in PBS; Vector Laboratories), followed by $1 \mathrm{~h}$ of incubation in the avidin-biotin-peroxydase complex solution (ABC solution; Vectastain, diluted 1:1000 in PBS; Vector Laboratories). Between each treatment, sections were thoroughly rinsed with PBS. The peroxydase complex was visualized after incubation for $10 \mathrm{~min}$ in a mix containing diaminobenzidine, ammonium chloride, ammonium sulfate, sodium acetate, glucose, and glucose oxydase. Sections were rinsed in sodium acetate $(2 \times 10 \mathrm{~min})$, mounted on gelatin-coated slides, dehydrated, and cover slipped. Quantification of the c-Fos immunoreactive cells was carried

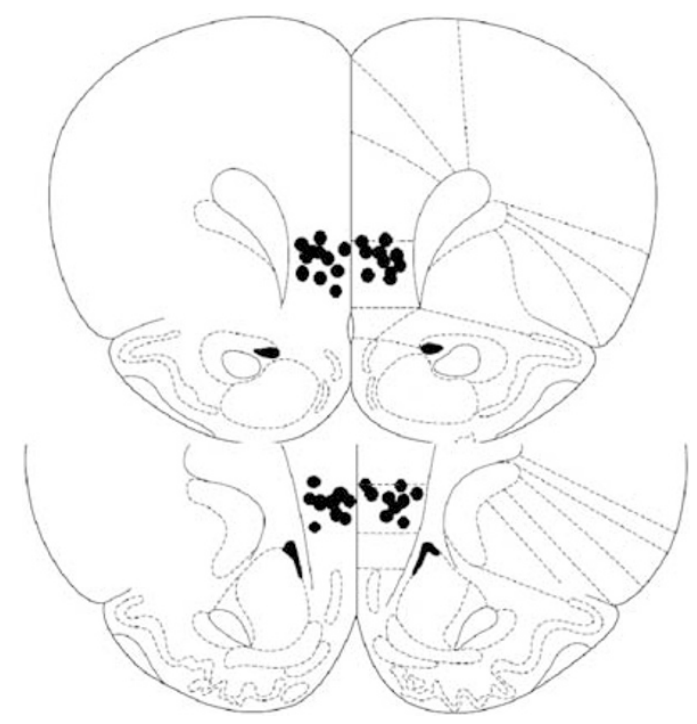

Figure I Schematic drawing illustrating the cannulae placements in the infralimbic cortex on coronal view at position +3.20 and $+2.70 \mathrm{~mm}$ anterior to Bregma (according to Paxinos and Watson, 1998).

out using a light microscope at a magnification of 100. Each section of interest was photographed using Nikon-ACT-1 software, and labeled cells were counted using the cell-count analysis ImageJ software. Fos-positive cells were automatically counted by the software according to a predetermined gray scale, shape, and size criteria. To maximize the measurement accuracy, we only took into account neurons that expressed a maximal level of Fos: only dark round or ovoid stains were counted as positive nuclei. Before each series of counting, we analyzed a reference slice containing a known number of c-Fos-labeled neurons to verify the settings of the microscope and software. Labeling was quantified bilaterally on 3 to 4 representative sections chosen to cover whole medial prefrontal cortex $(+3.20$ and $+2.70 \mathrm{~mm}$ anterior to bregma according to Paxinos and Watson, 1998). Fos-positive cells were counted on a surface representing $0.85 \mathrm{~mm}^{2}$. Results were expressed as the number of Fos-positive nuclei per square $\mathrm{mm}$.

\section{Statistics}

Differences were determined using mixed ANOVA. When appropriate, all the ANOVAs were followed by post hoc comparisons using least significant difference (LSD) multiple comparison tests. All tests were two tailed and a $P$-value of $<0.05$ was considered statistically significant.

\section{RESULTS}

\section{Anisomycin and Lidocaine into the IL Impaired Extinction Consolidation of Recent and Remote Fear Memory}

Rats were trained in CFC paradigm and were tested (T1) either 1 or 28 days later in order to assess recent and remote memory, respectively. In each condition, animals received vehicle, lidocaine, or anisomycin immediately after T1 (Figure 2a and b). There was no significant difference 
Extinction of recent fear memory

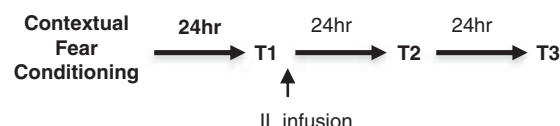

C

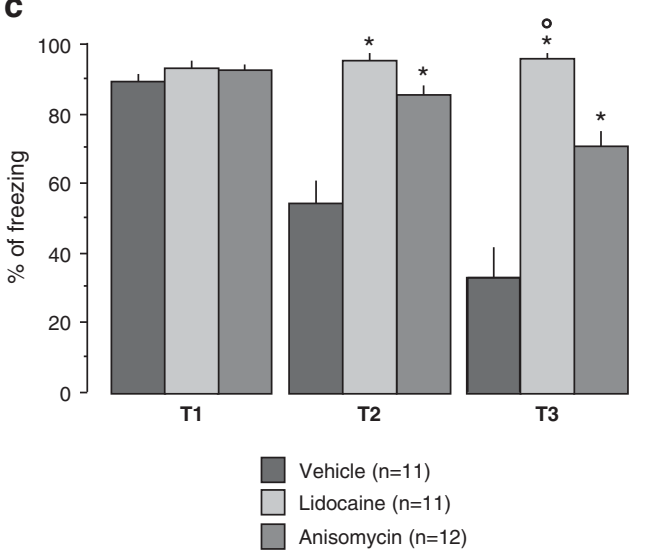

b

Extinction of remote fear memory

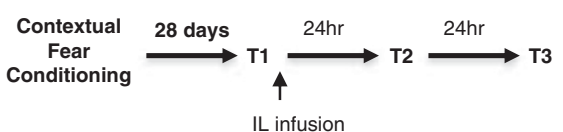

d

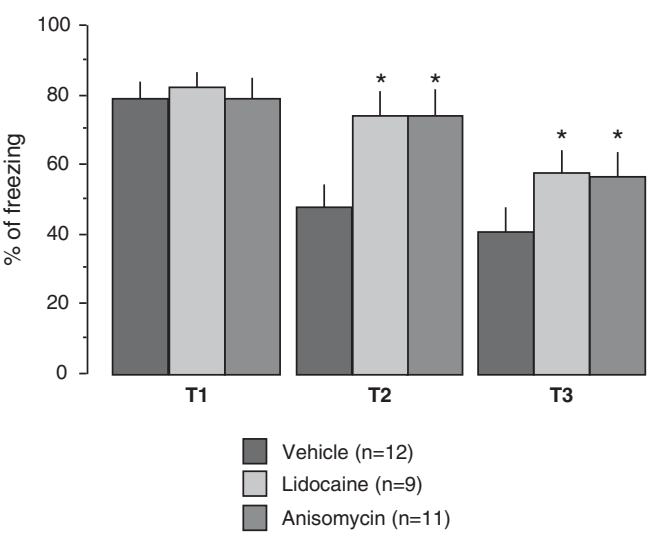

Figure 2 Infusions of lidocaine and anisomycin into the infralimbic cortex (IL) block extinction consolidation of recent and remote contextual fear memory. (a, b) Schematic representation of the experimental protocol used for assessing extinction of recent (a) and remote (b) contextual fear memory. (c, d) Behavioral results of animals infused into the IL with vehicle, lidocaine, or anisomycin immediately after TI, performed either I day (recent, c) or 28 days (remote, $d$ ) after conditioning. Intra-IL infusion of lidocaine and anisomycin impaired extinction of both recent and remote fear memory. *Significantly different from the corresponding vehicle-treated group $(P<0.05)$; ${ }^{\circ}$ significantly different from anisomycin-treated group on T3 $(P<0.05)$.

a

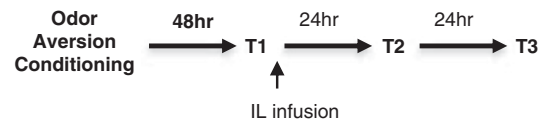

C

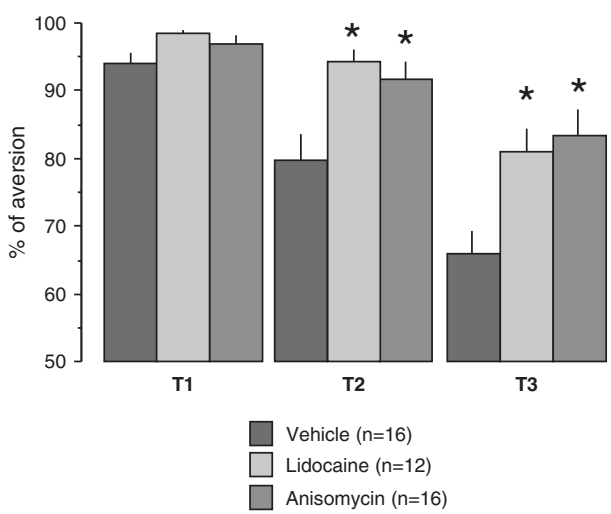

b

Extinction of remote aversion memory

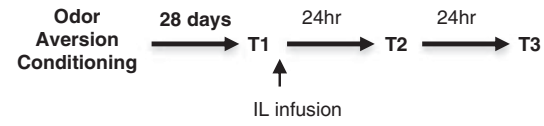

d

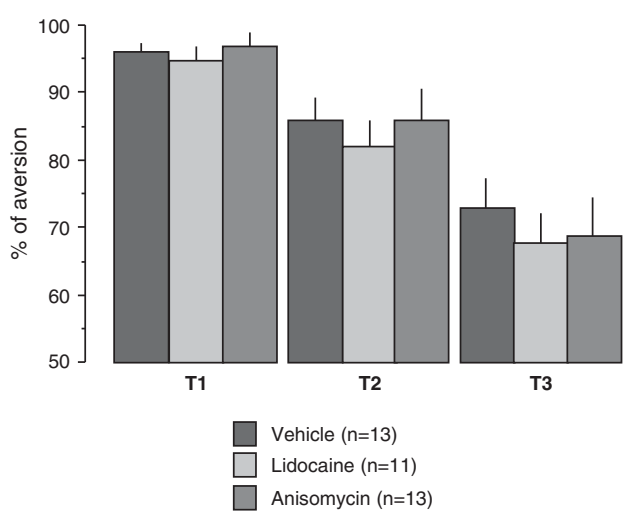

Figure 3 Infusions of lidocaine and anisomycin into the infralimbic cortex (IL) impair extinction consolidation of recent, but not remote, odor aversion memory. (a, b) Schematic representation of the experimental protocol used for assessing extinction of recent (a) and remote (b) odor aversion memory. (c, d) Behavioral results of animals infused into the IL with vehicle, lidocaine, or anisomycin immediately after TI, performed either 2 days (recent, c) or 28 days (remote, $d$ ) after conditioning. Intra-IL infusion of lidocaine and anisomycin impaired extinction of recent aversion memory but did not affect extinction of remote aversion memory. *Significantly different from the corresponding vehicle-treated group $(P<0.05)$.

between the groups on T1, suggesting comparable freezing on baseline $\left(\mathrm{F}_{1,60}<1\right.$; Figure $2 \mathrm{c}$ and $\left.\mathrm{d}\right)$. Three-way ANOVA (Drug $\times$ Age of Memory) with repeated measures (testing days, T2 and T3) showed significant effects of Drug $\left(\mathrm{F}_{2,60}=18.5\right.$, $P<0.001$; Figure $2 \mathrm{c}$ and $\mathrm{d})$, Age of Memory $\left(\mathrm{F}_{1,60}=6.1\right.$, $P<0.05)$, and Testing Days $\left(\mathrm{F}_{1,60}=56.5, P<0.001\right)$, and significant interactions of Drug $\times$ Age of Memory $\left(F_{2,64}=4.9\right.$, $P<0.01)$ and Drug $\times$ Testing day $\times$ Age of Memory $\left(\mathrm{F}_{2,64}=4.9\right.$,
$P<0.01)$. To better understand the source of the interactions, follow-up analyses were performed on recent and remote memory separately. In recent memory (Figure $2 \mathrm{c}$ ), there was a significant effect of Drug $\left(\mathrm{F}_{2,31}=33.9, P<0.001\right)$, Testing Days $\left(\mathrm{F}_{1,31}=45.1, P<0.001\right)$, and interaction between Drug and Testing Day $\left(\mathrm{F}_{2,31}=14.2, P<0.001\right)$. The post hoc analysis showed that the Drug-treated groups significantly differed from the Vehicle-treated groups $(P<0.001$ for both). However, 


\section{a Retrieval of recent and remote aversion memory}

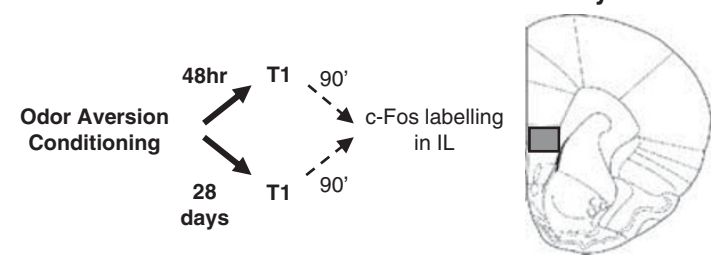

b
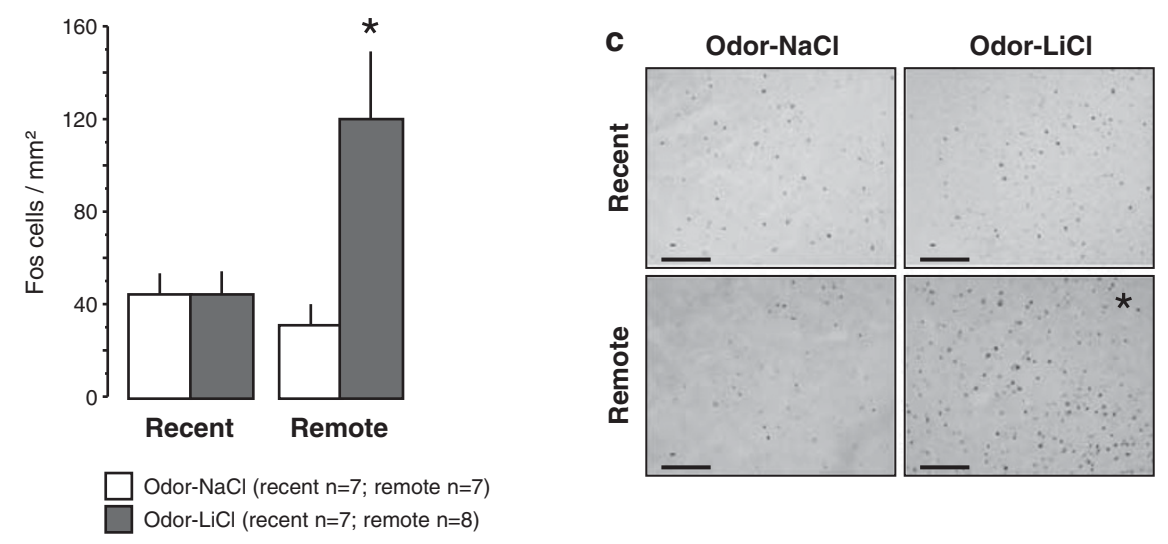

Figure 4 Enhancement of c-Fos labeling in the infralimbic cortex (IL) after retrieval of remote, but not recent, conditioned aversion memory. (a) Schematic representation of the experimental protocol used for assessing retrieval of recent and remote odor aversion memory and coronal brain sections adapted from Paxinos and Watson (1998), depicting in gray the regions in which Fos expression was analyzed. (b) Number of c-Fos-positive cells into the IL of animals killed 90 min after retrieval performed either 2 days (recent) or 28 days (remote) after conditioning (odor-LiCl) or pseudo-conditioning (odor-NaCl). Remote retrieval significantly enhanced c-Fos activation of the IL in comparison with recent retrieval and pseudoconditioned groups. (c) Representative pictures for c-Fos labeling in IL following recent or remote of odor aversion memory in conditioned (odor-LiCl) and pseudoconditioned (odor-NaCl) groups (scale bars $=200 \mu \mathrm{m})$. *Significantly different from all the other groups $(P<0.05)$. A full colour version of this figure is available at the Neuropsychopharmacology journal online.

the anisomycin and lidocaine groups also significantly differed from each other, with the lidocaine group showing enhanced freezing on T3 $(P<0.05)$. In remote memory (Figure $2 \mathrm{~d})$, ANOVA showed significant effect of Drug $\left(\mathrm{F}_{2,29}=13.7\right.$, $P<0.05)$ and Testing Days $\left(\mathrm{F}_{1,29}=19.1, P<0.001\right)$, but without significant interaction $\left(\mathrm{F}_{2,29}<1\right)$. The post hoc analysis showed that the Drug-treated groups significantly differed from the Vehicle-treated groups $(P<0.05$ for both), but did not differ from each other $(P>0.1)$. These results show that the IL is involved in mediating consolidation of extinction of recent and remote fear memories.

\section{Anisomycin and Lidocaine into the IL Impaired Extinction Consolidation of Recent, But Not Remote, Odor Aversion Memory}

Rats were trained for conditioned odor aversion and tested (T1) either 2 or 28 days after conditioning for recent and remote memory, respectively. In each condition, animals received vehicle, lidocaine, or anisomycin immediately after T1 (Figure $3 \mathrm{a}$ and $\mathrm{b}$ ). Univariate ANOVA on T1 did not show significant effects of Drug $\left(\mathrm{F}_{2,75}<1\right)$, Age of Memory $\left(\mathrm{F}_{1,75}<1\right)$, or interaction $\left(\mathrm{F}_{2,75}<1\right)$, suggesting comparable aversion index of all tested groups in recent and remote memory (Figure $3 c$ and $d$ ). Three-way ANOVA (Drug $\times$ Age of Memory) with repeated measures (Testing days, T2 and T3) showed significant effects of Testing Days $\left(\mathrm{F}_{1,75}=109\right.$, $P<0.001)$ and significant interaction between Drug and Age of Memory $\left(\mathrm{F}_{2,75}=6.2, P<0.01\right)$, suggesting that groups behaved differently according to the age of memory. There was no significant effect of Drug $\left(\mathrm{F}_{2,75}=1.9, P>0.1\right)$ or Age of Memory $\left(\mathrm{F}_{1,75}<1\right)$. To understand the source of interaction, recent and remote memory conditions were analyzed separately. In recent memory condition (Figure 3c), ANOVA for repeated measures showed significant effects of Drug $\left(\mathrm{F}_{2,41}=7.4, P<0.001\right)$ and Testing Days $\left(\mathrm{F}_{1,41}=75.9\right.$, $P<0.001)$ but without significant interaction $\left(F_{2,41}<1\right)$. The post hoc analysis showed that the Drug-treated groups significantly differed from the vehicle-treated group $(P<0.01$ for both), but they did not differ from each other. In remote memory condition (Figure $3 \mathrm{~d}$ ), there was only a significant effect of Testing Days $\left(\mathrm{F}_{1,34}=64.4, P<0.001\right)$ without significant effect of Drug or interaction $\left(F_{2,34}<1\right)$, suggesting that the groups extinguished similarly. Together, the results show that manipulations of the IL following retrieval affect extinction consolidation of recent COA memory, leaving remote memory intact.

\section{Expression of Remote, But Not Recent, Aversion Memory Induced IL Activation}

Results of the previous experiment suggest that retrieval of recent and remote $\mathrm{COA}$ memories differentially recruit the IL for extinction learning. The aim of the next experiment was to address the pattern of IL activation induced by retrieval of recent and remote COA memory (Figure 4a). For this purpose, we assessed c-Fos neuronal activation in the IL at $90 \mathrm{~min}$ after odor presentation either 2 or 28 days after 

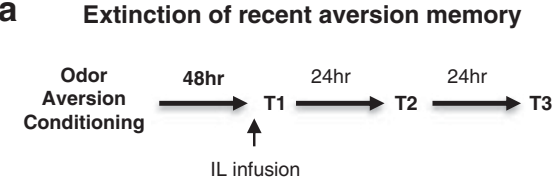

C

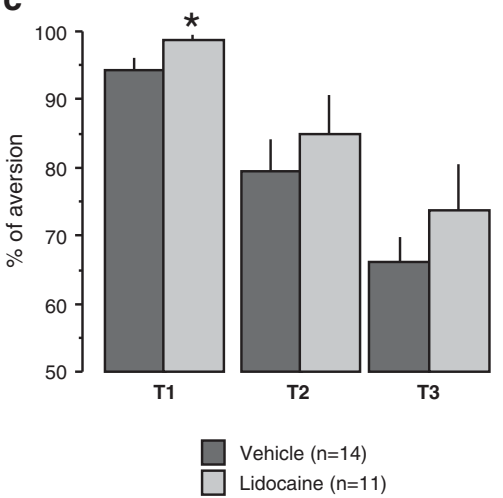

b
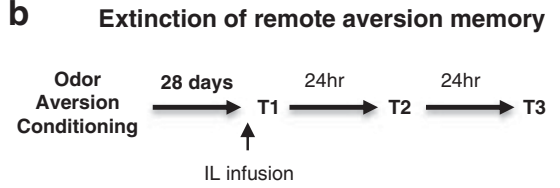

d

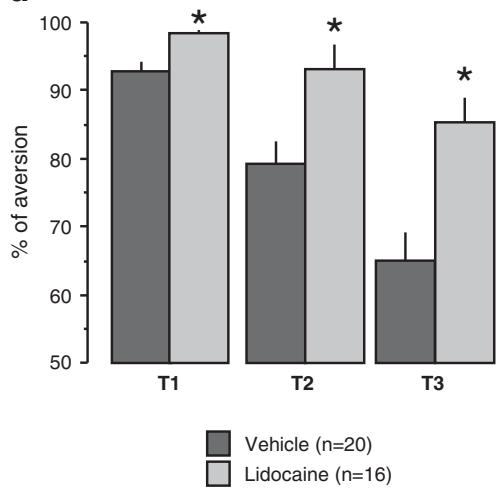

Figure 5 Infusion of lidocaine into the infralimbic cortex (IL) before retrieval impairs extinction learning of remote, but not recent, odor aversion memory. (a, b) Schematic representation of the experimental protocol used for assessing extinction of recent (a) and remote (b) odor aversion memory. (c, d) Behavioral results of animals infused into the IL with vehicle or lidocaine immediately before TI, performed either 2 days (recent, c) or 28 days (remote, d) after conditioning. Intra-IL infusion of lidocaine impaired extinction of remote aversion memory but did not affect extinction of recent aversion memory. * Significantly different from the corresponding vehicle-treated group $(P<0.05)$.

conditioning (odor- $\mathrm{LiCl}$ pairing) or pseudoconditioning (odor- $\mathrm{NaCl}$ pairing). During odor presentation, all conditioned animals drank $<4 \mathrm{ml}$ of odorized water, whereas all pseudoconditioned rats drank $>12 \mathrm{ml}$ (data not shown). There was a highly significant effect of Conditioning $(\mathrm{LiCl}$ versus $\mathrm{NaCl} ; \mathrm{F}_{1,25}=317.7, P<0.0001$ ), a significant effect of Age of Memory $\left(\mathrm{F}_{1,25}=4.2, P=0.05\right)$, and no interaction $\left(\mathrm{F}_{1,25}<1\right)$, indicating similar odor aversion whatever the age of the reactivated memory. Analyses of the c-Fos expression in IL showed significant effect of Conditioning $\left(\mathrm{F}_{1,25}=6.3\right.$, $P<0.02)$, a marginal effect of Age of Memory $\left(\mathrm{F}_{1,25}=3.1\right.$, $P=0.09)$, but a significant interaction between Conditioning and Age of Memory $\left(\mathrm{F}_{1,25}=6.4, P<0.02\right.$; Figure $4 \mathrm{~b}$ and $\left.\mathrm{c}\right)$. Further analysis indicates that the conditioned-remote group showed significantly higher c-Fos activation in the IL than all the other groups $(P<0.01)$. Conditioned-recent group was not significantly different from the pseudoconditioned groups $(P>0.1)$.

\section{Inactivation of the IL before Retrieval Impaired Extinction Memory of Remote, But Not Recent, COA}

The results of immunohistochemistry indicate a stronger IL activation during remote COA retrieval. This could suggest a faster recruitment of the IL in extinction processes when memory ages. To assess this possibility, animals were microinjected with lidocaine into the IL just before COA retrieval (T1) performed either 2 or 28 days after conditioning (Figure $5 a$ and $b$ ).

On T1, there was a significant effect of Drug $\left(\mathrm{F}_{1,53}=15.5\right.$, $P<0.001)$ but no effect of Age of Memory or interaction $\left(\mathrm{F}_{1,53}<1\right)$, indicating that lidocaine infusion into IL enhanced odor aversion regardless of the age of the reactivated memory (Figure $5 \mathrm{c}$ and $\mathrm{d}$ ). On the following tests, three-way ANOVA (Drug $\times$ Age of Memory) with repeated measures (Testing days, T2 and T3) showed significant effects of Drug $\left(\mathrm{F}_{1,54}=12.2, \quad P<0.005\right), \quad$ Testing Days $\quad\left(\mathrm{F}_{1,54}=153\right.$, $P<0.005)$, and significant interaction between Drug $\times$ Age of Memory $\times$ Testing Day $\left(\mathrm{F}_{1,54}=4.1, P<0.05\right)$. To understand this interaction, we then restricted the analysis to each age of memory. On recent memory (Figure $5 \mathrm{c}$ ), the results showed an effect of Testing Days $\left(\mathrm{F}_{1,23}=70.2, P<0.005\right)$ indicating extinction but no effect of Drug or interaction $\left(\mathrm{F}_{1,23}<1\right)$. However, on remote memory (Figure $5 \mathrm{~d}$ ), there was a significant effect of Testing Days $\left(\mathrm{F}_{1,35}=54.1, P<0.005\right)$, Drug $\left(\mathrm{F}_{1,35}=10.5, P<0.005\right)$, and interaction between Drug and Testing Days $\left(\mathrm{F}_{1,35}=15.5, P<0.005\right)$. Further analysis shows that there was a significant difference between the groups on the two testing days $(P<0.05)$, with the group infused with lidocaine showing slower COA extinction. This indicates that preretrieval manipulations of the IL of remote, but not recent, COA memory impaired extinction memory.

\section{DISCUSSION}

Using either reversible inactivation or protein synthesis inhibitor targeting the postretrieval consolidation phase (Akirav et al, 2006a; Eisenberg et al, 2003), we showed that the IL is involved in extinction consolidation of both recent and remote CFC as was recently shown (Rosas-Vidal et al, 2014). Targeting the same time window, the results show that the IL is involved only in extinction consolidation of recent, but not remote, COA. Using c-Fos, the neuronal activation marker we show that remote, but not recent, COA memory reactivation induced IL activation, and preretrieval inactivation of the IL impaired extinction of remote, but not recent, COA. These data show that IL has a role in the mediation of COA extinction, indicating that this brain area is involved in the extinction of fear and nonfear-based associations 
(Akirav et al, 2006a, b; Maroun et al, 2012; Milad and Quirk, 2002; Peters et al, 2009; Slouzkey et al, 2013) and suggest a dissociation in the engagement of the IL in learning and consolidation of COA extinction over time.

Our results indicate a similar requirement of the IL in the consolidation of recent and remote fear extinction and join the recent findings reported by Rosas-Vidal et al (2014) who showed similar effect of intra-IL microinfusion of brainderived neurotrophic factor (BDNF) in recent (1 day after conditioning) and remote (14 days after conditioning) extinction of fear memories. This corroborates previous results that we and others obtained regarding the crucial role of the IL subregion of the mPFC in the mediation of consolidation of recent fear extinction (Milad et al, 2004; Kritman and Maroun, 2012; Laurent and Westbrook, 2009; Sierra-Mercado et $a l, 2010$ ) and extends it to remote fear memory. Although previous studies indicated that remote fear extinction can affect activation and lead to structural changes in the IL (Vetere, et al, 2011; Vetere, et al, 2012), there was neither direct assessment for the role of the IL in extinction of remote memories nor direct comparison of the IL involvement in recent and remote fear extinction. Our results suggest a permanent role of the IL in the consolidation of fear extinction that is not limited to the age of the memory (see also Rosas-Vidal et al, 2014). It should be noted however that there is a difference between the kinetics of fear extinction in the treated groups in recent as compared with remote fear extinction. Although IL treatment after the recent memory retrieval blocked extinction from T2 to T3, the treated groups in the remote memory showed some levels of extinction from T2 to T3. This could be because of weaker fear memory in the remote condition (80\% versus $90-95 \%$ of freezing, see Figure $2 \mathrm{c}$ and $\mathrm{d}$ ) as previous work indicated that the strength of the memory during retrieval session clearly determine the effect of pharmacological treatment (Eisenberg et al, 2003; Suzuki et al, 2004).

The results of the present study also indicate differential requirement of the IL in the consolidation of recent and remote $\mathrm{COA}$ extinction. Concerning recent extinction, the results obtained here with olfactory stimulation extend our previous study obtained with gustatory stimulation, ie, conditioned taste aversion (Akirav et al, 2006a; Maroun et al, 2012; Shehadi and Maroun, 2013), indicating the crucial role of the IL in the consolidation of recent aversion extinction whatever the chemosensory information involved. More importantly, the absence of IL involvement in extinction consolidation of remote memory indicates that the circuitry controlling the consolidation of aversion extinction reorganizes over time in a different pattern than that of CFC. Numerous structures like the basolateral amygdala, the hippocampus, the perirhinal, and insular cortices have been shown to participate in the extinction consolidation of flavor aversion (Bahar et al, 2003; Garcia-Delatorre et al, 2010). These results suggest that COA extinction memory may become broadly distributed across multiple circuits with increasing time from acquisition (Nadel and Moscovitch, 1997), explaining why the IL is not necessary for extinction consolidation of remote COA memory. However, our results do not completely exclude the IL from the network involved in remote COA extinction but suggest a different time course for the recruitment of the IL. Indeed, earlier inactivation of the IL at the preretrieval session impaired subsequent extinction of remote COA, suggesting a faster recruitment of the IL with a shift from consolidation to learning of extinction over time. Similar faster recruitment was previously suggested for the hippocampus concerning remote memory (Debiec et al, 2002; Goshen et al, 2011). Altogether, these results point to an important difference between CFC and COA regarding the role of the IL in consolidation of remote memory extinction. Major differences between the two paradigms concern the dependency on hippocampus and amygdala. Although CFC clearly relies on hippocampus even at remote time points (Debiec et al, 2002; Einarsson et al, 2014; Einarsson and Nader, 2012; Goshen et al, 2011), cue-based (taste and odor) food aversion memory is considered hippocampal independent (Ballarini, et al, 2009; Cui et al, 2005; Ding et al, 2008; Gusev et al, 2005; Josselyn et al, 2004). However, this assumption was recently challenged for conditioned taste aversion (Chinnakkaruppan et al, 2014) and long time ago for COA (Bermudez-Rattoni et al, 1986; Miller et al, 1986). Concerning the amygdala, recent results suggest that the systems mediating context fear are reorganized within 2 weeks of learning, with a significant reduction in the percentage of reactivated neurons in the basolateral amygdala during the remote memory test (Tayler et al, 2012). However, we demonstrated that manipulations of basolateral amygdala induced a more important effect on remote than on recent COA memory retrieval (Sevelinges et al, 2009b). According to its role in taste aversion extinction (Akirav and Maroun, 2007; Bahar et al, 2003), the basolateral amygdala could therefore play an increasing role in extinction consolidation of COA over time.

It seems counteractive that preretrieval microinfusion of lidocaine in COA for recent memories had no effect on retention on subsequent days, whereas postretrieval microinfusions had effects on the subsequent testing days. However, we tend to believe that this could be attributable to the dose of lidocaine used here that induced a complete inhibition of neural activity within 5 min after infusion that lasts for 15-30 min (Tehovnik and Sommer, 1997; Lomber, 1999; Boehnke and Rasmusson, 2001). Thus, IL infusion of lidocaine before COA retrieval was thought to induce a functional inactivation of the IL that was limited to the retrieval session lasting $20 \mathrm{~min}$ and did not extend to postretrieval consolidation phase. It should be noted that inactivation of the IL before the retrieval session, which is also the initiation of extinction session (Berman and Dudai, 2001; Eisenberg et al, 2003; Akirav et al, 2006b; Slouzkey et al, 2013), enhanced both recent and remote COA expression, probably because of a blockade of within-session extinction consistent with previous findings obtained in CFC following pre-test IL inactivation (Laurent and Westbrook, 2009; SierraMercado et al, 2010). Contrary to these previous CFC studies, it is worthy to note that our test of COA retrieval cannot be divided into trials. Thus, we cannot differentiate between expression or within-session extinction.

However, only in the remote memory condition, subsequent extinction was impaired as the kinetic of extinction was intact in the recent memory condition. Interestingly, monitoring c-Fos activation in the IL showed an increase in activation $90 \mathrm{~min}$ following retrieval of remote COA, as previously shown for CFC (Frankland et al, 2004) that could not be attributed to retrieval by itself, as there is no such 
activation in the recent memory condition, but rather to retrieval-induced extinction. The finding that the role of IL may shift from recall to extinction over time fits with recent data about the role of the ventral part of the MPFC in recall and extinction of cocaine-associated memory (Van den Oever et al, 2013). Using optogenetic tools the authors found that the activity of the ventral mPFC pyramidal cells is implicated in the recall of recent cocaine-associated memory but also engaged in its remote extinction (Van den Oever et al, 2013).

Blockade of protein synthesis after recent COA retrieval impaired consolidation of extinction memory. Therefore, the lack of c-Fos protein induction in IL by retrieval of recent COA memory indicates that c-Fos is not one of the obligatory proteins that have to be newly formed for consolidation of extinction memory. Indeed, other proteins have been identified as being important in IL for consolidation of recent aversion memory extinction like coffilin and BDNF (Wang et al, 2013; Xin et al, 2014). Another possibility that could explain the lack of c-Fos induction in IL by recent $\mathrm{COA}$ retrieval could be related to the absence of analyses of subtypes of cells. For instance, taste stimulation did not induce general c-Fos in the gustatory cortex but, when the different subtypes of cells were differentiated, a specific activation of c-Fos in GABAergic neurons was reported (Doron and Rosenblum, 2010). This suggests that different subtypes of neurons participate in a given phase to control the behavioral output, as recently indicated by Van den Oever et al (2013).

Upon retrieval of the original memory that was formed during the association, two processes compete to dominate: extinction and reconsolidation. We tend however to favor an effect on extinction rather than reconsolidation as we showed here a blockade rather than a facilitation of extinction memory. Treatments like protein synthesis inhibitors targeting the reconsolidation phase are expected to weaken the original association that may result in reduction in freezing/aversion and facilitate extinction memory (Eisenberg et al, 2003; Suzuki et al, 2004). Interestingly, two recent studies indicate that dorsal mPFC (anterior cingulate cortex and prelimbic cortex) subserves recent and remote contextual fear memory reconsolidation (Einarsson and Nader, 2012), suggesting a dissociation and maybe a competition between the ventral part of the mPFC, involved in extinction, and the dorsal mPFC, involved in reconsolidation. Whether similar dissociation, ie, specific involvement of the dorsal $\mathrm{mPFC}$ in reconsolidation processes, exists in nonfear-based association like COA remains to be established over time.

The results clearly show that extinction of different types of aversive memories do not have similar engagement of the brain circuits, especially not that of the IL, and that extinction of remote memories of COA could be distributed in other circuits than the IL. Our findings emphasize that remote aversive odor memories could be distributed in wider brain circuits. Memory for odors that are associated with intense emotional experiences is often strongly engraved and resistant to decay over long intervals (Sullivan et al, 2000). Similarly, it was previously suggested that odors are more likely to affect memory than other sensory modalities or experiences (Engen 1973; Chu and Downes, 2000; Kadohisa, 2013). Furthermore, the robustness of trauma-related smells was shown to trigger symptoms in patients with posttraumatic stress disorders (see, eg, Vermetten and Bremner, 2003).

In conclusion, our findings show dissociation of the requirement of the IL in consolidation of recent versus remote extinction memory of two different emotional paradigms, $\mathrm{CFC}$ and COA, and a double dissociation in its involvement in acquisition versus consolidation of COA extinction. Future studies should further elucidate activity in this region to better understand its role in the extinction of the two types of memory over time. Altogether, our results extend our knowledge on the role played by the IL in extinction memory and show it is more complex than first believed.

\section{FUNDING AND DISCLOSURE}

The authors declare no conflict of interest.

\section{ACKNOWLEDGMENTS}

We thank J Sauvant and P Trifilieff for technical assistance. This work was supported by the Israel Science Foundation grant (613/13 to MM) and the grant Emergence de Jeune Equipe INRA (to GF).

\section{REFERENCES}

Akirav I, Segev A, Motanis H, Maroun M (2009). D-cycloserine into the BLA reverses the impairing effects of exposure to stress on the extinction of contextual fear, but not conditioned taste aversion. Learn Mem 16: 682-686.

Akirav I, Maroun M (2007). The role of the medial prefrontal cortex-amygdala circuit in stress effects on the extinction of fear. Neural Plast 2007.

Akirav I, Khatsrinov V, Vouimba RM, Merhav M, Ferreira G, Rosenblum $\mathrm{K}$ et al (2006a). Extinction of conditioned taste aversion depends on functional protein synthesis but not on NMDA receptor activation in the ventromedial prefrontal cortex. Learn Mem 13: 254-258.

Akirav I, Raizel H, Maroun M (2006b). Enhancement of conditioned fear extinction by infusion of the GABA(A) agonist muscimol into the rat prefrontal cortex and amygdala. Eur $J$ Neurosci 23: 758-764.

Bahar A, Samuel A, Hazvi S, Dudai Y (2003). The amygdalar circuit that acquires taste aversion memory differs from the circuit that extinguishes it. Eur J Neurosci 17: 1527-1530.

Ballarini F, Moncada D, Martinez MC, Alen N, Viola H (2009). Behavioral tagging is a general mechanism of long-term memory formation. Proc Natl Acad Sci U S A 106: 14599-14604.

Berman DE, Dudai Y (2001). Memory extinction, learning anew, and learning the new: dissociations in the molecular machinery of learning in cortex. Science 291: 2417-2419.

Bermudez-Rattoni F, Grijalva CV, Kiefer SW, Garcia J (1986). Flavor-illness aversions: the role of the amygdala in the acquisition of taste-potentiated odor aversions. Physiol Behav 38: 503-508.

Blanchard DC, Blanchard RJ (1972). Innate and conditioned reactions to threat in rats with amygdaloid lesions. J Comp Physiol Psychol 81: 281-290.

Boehnke SE, Rasmusson DD (2001). Time course and effective spread of lidocaine and tetrodotoxin delivered via microdialysis: an electrophysiological study in cerebral cortex. J Neurosci Methods 105: 133-141.

Chapuis J, Messaoudi B, Ferreira G, Ravel N (2007). Importance of retronasal and orthonasal olfaction for odor aversion memory in rats. Behav Neurosci 121: 1383. 
Chinnakkaruppan A, Wintzer ME, McHugh TJ, Rosenblum K (2014). Differential contribution of hippocampal subfields to components of associative taste learning. J Neurosci 34: 11007-11015.

Chu S, Downes JJ (2000). Long live Proust: The odour-cued autobiographical memory bump. Cognition 75: B41-B50.

Corcoran KA, Leaderbrand K, Radulovic J (2013). Extinction of remotely acquired fear depends on an inhibitory NR2B/PKA pathway in the retrosplenial cortex. J Neurosci 33: 19492-19498.

Cui Z, Lindl KA, Mei B, Zhang S, Tsien JZ (2005). Requirement of NMDA receptor reactivation for consolidation and storage of nondeclarative taste memory revealed by inducible NR1 knockout. Eur J Neurosci 22: 755-763.

Debiec J, LeDoux JE, Nader K (2002). Cellular and systems reconsolidation in the hippocampus. Neuron 36: 527-538.

Desgranges B, Sevelinges Y, Bonnefond M, Levy F, Ravel N, Ferreira G (2009). Critical role of insular cortex in taste but not odour aversion memory. Eur J Neurosci 29: 1654-1662.

Ding HK, Teixeira CM, Frankland PW (2008). Inactivation of the anterior cingulate cortex blocks expression of remote, but not recent, conditioned taste aversion memory. Learning \& Memory 15: 290-293.

Doron G, Rosenblum K (2010). c-Fos expression is elevated in GABAergic interneurons of the gustatory cortex following novel taste learning. Neurobiol Learn Mem 94: 21-29.

Einarsson E, Pors J, Nader K (2014). Systems reconsolidation reveals a selective role for the anterior cingulate cortex in generalized contextual fear memory expression. Neuropsychopharmacology 40: 480-487.

Einarsson EÖ, Nader K (2012). Involvement of the anterior cingulate cortex in formation, consolidation, and reconsolidation of recent and remote contextual fear memory. Learning \& Memory 19: 449-452.

Eisenberg M, Kobilo T, Berman DE, Dudai Y (2003). Stability of retrieved memory: inverse correlation with trace dominance. Science 301: 1102-1104.

Engen T (1973). The sense of smell. Annu Rev Psychol 24: 187-206.

Frankland PW, Bontempi B (2005). The organization of recent and remote memories. Nat Rev Neurosci 6: 119-130.

Frankland PW, Bontempi B, Talton LE, Kaczmarek L, Silva AJ (2004). The involvement of the anterior cingulate cortex in remote contextual fear memory. Science 304: 881-883.

Garcia-Delatorre P, Rodriguez-Ortiz CJ, Balderas I, Bermudez-Rattoni F (2010). Differential participation of temporal structures in the consolidation and reconsolidation of taste aversion extinction. Eur J Neurosci 32: 1018-1023.

Goshen I, Brodsky M, Prakash R, Wallace J, Gradinaru V, Ramakrishnan C et al (2011). Dynamics of retrieval strategies for remote memories. Cell 147: 678-689.

Gusev PA, Cui C, Alkon DL, Gubin AN (2005). Topography of Arc/ Arg3.1 mRNA expression in the dorsal and ventral hippocampus induced by recent and remote spatial memory recall: dissociation of CA3 and CA1 activation. J Neurosci 25: 9384-9397.

Inda MC, Muravieva EV, Alberini CM (2011). Memory retrieval and the passage of time: from reconsolidation and strengthening to extinction. J Neurosci 31: 1635-1643.

Josselyn S, Kida S, Silva A (2004). Inducible repression of CREB function disrupts amygdala-dependent memory. Neurobiol Learn Mem 82: 159-163.

Kadohisa M (2013). Effects of odor on emotion, with implications. Frontiers Sys Neurosci 7: 66.

Kim JJ, Fanselow MS, DeCola JP, Landeira-Fernandez J (1992). Selective impairment of long-term but not short-term conditional fear by the N-methyl-D-aspartate antagonist APV. Behav Neurosci 106: 591.

Knowlton BJ, Fanselow MS (1998). The hippocampus, consolidation and on-line memory. Curr Opin Neurobiol 8: 293-296.

Kritman M, Maroun M (2012). Inhibition of the PI3 kinase cascade in corticolimbic circuit: temporal and differential effects on contextual fear and extinction. Inter J Neuropsychopharmacol 1: $1-9$.

Lahoud N, Maroun M (2013). Oxytocinergic manipulations in corticolimbic circuit differentially affect fear acquisition and extinction. Psychoneuroendocrinology 38: 2184-2195.

Laurent V, Westbrook RF (2009). Inactivation of the infralimbic but not the prelimbic cortex impairs consolidation and retrieval of fear extinction. Learn Mem 16: 520-529.

Lomber SG (1999). The advantages and limitations of permanent or reversible deactivation techniques in the assessment of neural function. J Neurosci Methods 86: 109-117.

Lopez J, Vasconcelos AP, Cassel JC (2008). Differential sensitivity of recent vs. remote memory traces to extinction in a water-maze task in rats. Neurobiol Learn Mem 90: 460-466.

Maroun M, Kavushansky A, Holmes A, Wellman C, Motanis H (2012). Enhanced extinction of aversive memories by highfrequency stimulation of the rat infralimbic cortex. PLoS One 7: e35853.

Milad MR, Quirk GJ (2002). Neurons in medial prefrontal cortex signal memory for fear extinction. Nature 420: 70-74.

Milad MR, Vidal-Gonzalez I, Quirk GJ (2004). Electrical stimulation of medial prefrontal cortex reduces conditioned fear in a temporally specific manner. Behav Neurosci 118: 389-394.

Miller JS, Nonneman AJ, Kelly KS, Neisewander JL, Isaac WL (1986). Disruption of neophobia, conditioned odor aversion, and conditioned taste aversion in rats with hippocampal lesions. Behav Neural Biol 45: 240-253.

Motanis H, Maroun M (2010). Exposure to a novel context following contextual fear conditioning enhances the induction of hippocampal long-term potentiation. Eur J Neurosci 32: 840-846.

Nadel L, Moscovitch M (1997). Memory consolidation, retrograde amnesia and the hippocampal complex. Curr Opin Neurobiol 7: 217-227.

Paxinos G, Watson C (1998). The Rat Brain in Stereotaxic Coordinates. Academic Press: San DiegoSan Diego.

Peters J, Kalivas PW, Quirk GJ (2009). Extinction circuits for fear and addiction overlap in prefrontal cortex. Learn Memory 16: 279-288.

Restivo L, Vetere G, Bontempi B, Ammassari-Teule M (2009). The formation of recent and remote memory is associated with timedependent formation of dendritic spines in the hippocampus and anterior cingulate cortex. J Neurosci 29: 8206-8214.

Rosas-Vidal LE, Do-Monte FH, Sotres-Bayon F, Quirk GJ (2014). Hippocampal-prefrontal BDNF and memory for fear extinction. Neuropsychopharmacology 39: 2161-2169.

Sevelinges Y, Desgranges B, Ferreira G (2009a). The basolateral amygdala is necessary for the encoding and the expression of odor memory. Learn Mem 16: 235-242.

Sevelinges Y, Levy F, Mouly AM, Ferreira G (2009b). Rearing with artificially scented mothers attenuates conditioned odor aversion in adulthood but not its amygdala dependency. Behav Brain Res 198: 313-320.

Shehadi K, Maroun M (2013). Different effects of low frequency stimulation to infralimbic prefrontal cortex on extinction of aversive memories. Brain Res 1490: 111-116.

Sierra-Mercado D, Padilla-Coreano N, Quirk GJ (2010). Dissociable roles of prelimbic and infralimbic cortices, ventral hippocampus, and basolateral amygdala in the expression and extinction of conditioned fear. Neuropsychopharmacology 36: $529-538$

Slotnick BM, Westbrook F, Darling FMC (1997). What the rat's nose tells the rat's mouth: long delay aversion conditioning with aqueous odors and potentiation of taste by odors. Learn Behav 25: 357-369.

Slouzkey I, Rosenblum K, Maroun M (2013). Memory of conditioned taste aversion is erased by inhibition of PI3K in the insular cortex. Neuropsychopharmacology 38: 1143-1153. 
Squire LR, Alvarez P (1995). Retrograde amnesia and memory consolidation: a neurobiological perspective. Curr Opin Neurobiol 5: $169-177$.

Sullivan RM, Landers M, Yeaman B, Wilson DA (2000). Neurophysiology: Good memories of bad events in infancy. Nature 407: 38-39.

Suzuki A, Josselyn SA, Frankland PW, Masushige S, Silva AJ, Kida S (2004). Memory reconsolidation and extinction have distinct temporal and biochemical signatures. J Neurosci 24: 4787-4795.

Tayler KK, Tanaka KZ, Reijmers LG, Wiltgen BJ (2012). Reactivation of neural ensembles during the retrieval of recent and remote memory. Curr Biol 23: 99-106.

Tehovnik EJ, Sommer MA (1997). Effective spread and timecourse of neural inactivation caused by lidocaine injection in monkey cerebral cortex. J Neurosci Methods 74: 17-26.

Van den Oever MC, Rotaru DC, Heinsbroek JA, Gouwenberg Y, Deisseroth K, Stuber GD et al (2013). Ventromedial prefrontal cortex pyramidal cells have a temporal dynamic role in recall and extinction of cocaine-associated memory. J Neurosci 33: $18225-18233$.
Vermetten E, Bremner JD (2003). Olfaction as a traumatic reminder in posttraumatic stress disorder: case reports and review. J Clin Psychiatry 64: 202-207.

Vetere G, Restivo L, Novembre G, Aceti M, Lumaca M, Ammassari-Teule M (2011). Extinction partially reverts structural changes associated with remote fear memory. Learn Mem 18: 554-557.

Vetere G, Restivo L, Ammassari-Teule M (2012). Pre-synaptic control of remote fear extinction in the neocortex. Front Behav Neurosci 6.

Wang Y, Dong Q, Xu XF, Feng X, Xin J, Wang DD et al (2013). Phosphorylation of cofilin regulates extinction of conditioned aversive memory via AMPAR trafficking. J Neurosci 33: 6423-6433.

Xin J, Ma L, Zhang TY, Yu H, Wang Y, Kong L et al (2014). Involvement of BDNF signaling transmission from basolateral amygdala to infralimbic prefrontal cortex in conditioned taste aversion extinction. J Neurosci 34: 7302-7313.

Zelikowsky M, Bissiere S, Fanselow MS (2012). Contextual fear memories formed in the absence of the dorsal hippocampus decay across time. J Neurosci 32: 3393-3397. 